\title{
THE LATE EFFECT OF THE HARELIP AND ITS CORREC- TION BY A NEW OPERATION
}

\author{
By J. L. Aymard, M.R.C.S., L.R.C.P. \\ Late Plastic Sungeon, Cambridge Hospital, Aldershot, Southampton, England; \\ Plastic Surgeon and Ear, Nose and Throat Specialist to the Queen's \\ Hospital for Facial Injuries
}

\begin{abstract}
$\mathrm{T}^{\mathrm{H}}$ HE photographs of this young girl are representative of a series of similar successful cases, and present a condition familiar not only to surgeons but the general public - the tightly stretched upper lip giving the appearance of the underhung jaw, the absence of the red margin, and the loss of the proper contour in both dimensions, together with the absence of the philtrum. The nose in such cases is very frequently flattened and deflected, and nasal stenosis a necessary accompaninient. In young ladies, especially, the appearance is detrimental
\end{abstract}

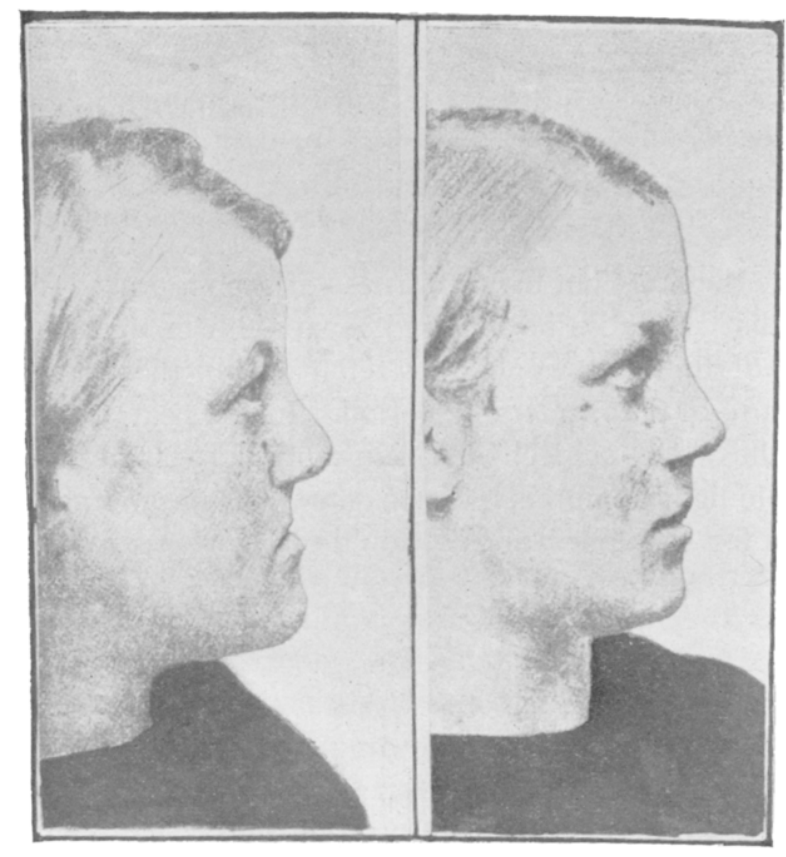

Fig. 1.

Fig. 2.

Fig. 1.-A typical side view before operation, giving the appearance of an underhung lower jaw. Fig. 2.-Side view after correction by operation.

to marriage, and as far as I know there are no cases on record in which the difficulty of correction has hitherto been overcome.

The nasal condition associated so frequently with these cases is extremely complicated, and many considerations must be taken in view before any attempt is made to correct the same. A study of the correct anatomy of these parts, published by me in the Journal of Anatomy, will assist considerably, but I would 
lay it down as a law that the preliminary correction of the nasal trouble and the lip must be made at the same time. To correct the position of the nose it is frequently necessary to readjust the nasal crest, and the complete exposure of the floor of the nose is often essential. Although the operation as illustrated looks comparatively simple and some may be tempted to try it for the sake of kudos, I would warn such surgeons to make themselves familiar with all the necessary adjuncts to success, such as the correct amount of overcorrection, the freeing of all the tissues in the neighborhood and the supporting sutures, and many other details known to the plastic surgeon. It is obviously a temptation to perform any operation for the restoration of the lip, and then to send the case to the nasal

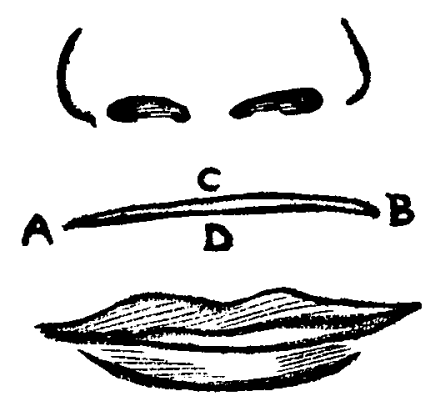

Fig. 3.

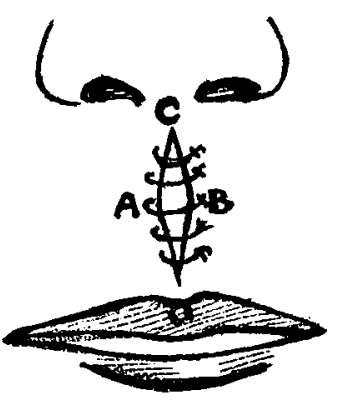

Fig. 4.

Fig. 3.- Shows the incision made from the inside through the whole thickness of the lip up to the inner

Fig. 4.- Shows the incision sewed up in the opposite direction. The deep supporting sutures are not shown, nor the incisions for freeing the surrounding tissues.

surgeon to restore the nose, but the unfairness of such a procedure will be obvious when I explain that if the lip be repaired separately the subsequent operation to expose the floor of the nose would so stretch the lip that it would break down even after the lapse of a considerable period. In the near future I hope to publish a paper on the whole subject of harelip and cleft-palate, which I think may throw considerable light upon this hitherto somewhat unsatisfactory subject. The period preferable for such operations I would place from six to four years of age. 